\title{
Diffusion Kinetics Analysis of Cation Diffusion in YSZ and LSGM
}

\author{
I.V. Belova ${ }^{1, a}$ and G.E. Murch ${ }^{1, b}$, D. Samuelis ${ }^{2, c}$ and M. Martin ${ }^{2, d}$ \\ ${ }^{1}$ Diffusion in Solids Group \\ Centre for Geotechnical and Materials Modelling \\ School of Engineering, University of Newcastle \\ Callaghan NSW 2308 Australia \\ ${ }^{2}$ Institute of Physical Chemistry \\ RWTH Aachen University \\ Landoltweg 2, 52056 Aachen Germany
}

\begin{abstract}
a Irina.Belova@newcastle.edu.au, ${ }^{\text {b }}$ Graeme.Murch@newcastle.edu.au, ${ }^{\mathrm{c}}$ samuelis@pc.rwthaachen.de, ${ }^{\mathrm{d}}$ martin@rwth-aachen.de
\end{abstract}

Keywords: cation diffusion, tracer diffusion, solid electrolytes, demixing, LSGM, YSZ

\begin{abstract}
In this paper, we discuss different possible cation diffusion mechanisms in YSZ and LSGM. Monte Carlo simulations are also reported of tracer diffusivities in LSGM for a postulated cluster mechanism. These simulations extend earlier simulations on un-doped material. The limits of the ratio of the diffusivities are consistent with experimental tracer diffusion findings over a wide range of cation-vacancy exchange frequencies. We also develop relationships between the phenomenological coefficients and use these relationships to predict possible demixing and interdiffusion experimental outcomes.
\end{abstract}

\section{Introduction}

Cation diffusion in the high anion conductivity electrolytes YSZ (yttria-stabilized zirconia) and LSGM (doped lanthanum gallate $\mathrm{La}_{(1-\mathrm{x})} \mathrm{Sr}_{\mathrm{x}} \mathrm{Ga}_{(1-\mathrm{y})} \mathrm{Mg}_{\mathrm{y}} \mathrm{O}_{(3-(\mathrm{x}+\mathrm{y}) / 2)}$ ) is important for understanding their synthesis and high temperature creep behaviour as well as their cation demixing behaviour after prolonged periods at high temperatures in an electric field.

In the case of YSZ, diffusion via independent cation vacancies, by vacancy-pairs, by vacancy triplets and higher order vacancy clusters has been analysed theoretically and by means of Monte Carlo simulation [1]. The implications of these mechanisms on demixing experiments on YSZ have been discussed at length [1]. We summarize these findings in this paper.

In the case of LSGM, tracer diffusivities of three cations ( $\mathrm{La}, \mathrm{Sr}$ and $\mathrm{Mg}$ ) have been measured. All three are nearly identical [2,3]. To explain these experimental findings a bound defect cluster mechanism containing cation vacancies of both the A- and the B- sublattice and anion vacancies was proposed recently by Schultz et al. [3] as being the principal vehicle for cation diffusion in LSGM. Four different cluster mechanisms are possible in the lanthanum gallate structure. As was pointed out in [4] only a cluster containing a single anion vacancy can produce long range diffusion. The other clusters would require partial dissociation of the anion vacancies. In this paper, Sum-rule expressions for the phenomenological coefficients are derived. Versions of these expressions had been tested by Monte Carlo computer simulation [4] for the case of undoped lanthanum gallate. The implications of the findings on possible demixing measurements and interdiffusion experiments on LSGM are discussed here in detail.

\section{Collective Diffusion in LSGM}

The cluster mechanism first proposed in [3] can be considered in the following way. We define possible vector-jumps of the $A$-type of cation vacancy (with an exchange with A or A' 
cation) in the given cluster as $\vec{r}_{A}^{1}$ and $\vec{r}_{A}^{2}$, of the B-type cation vacancy (with an exchange with B or B' cation) as $\vec{r}_{B}$, and the $O$ vacancy as $\vec{r}_{O}^{1}$ and $\vec{r}_{O}^{2}$ (see Fig. 1 ). For the mixed oxide $A_{1-x} A_{x}{ }^{\prime} B_{1-}$ ${ }_{y} B_{y}^{\prime} O_{3-(x+y) / 2}$ (here $A$ is lanthanum, $A^{\prime}$ is strontium, $B$ is gallium and $B^{\prime}$ is magnesium) we will have the condition that $A$ and $A^{\prime}$ cations share the same sublattice and can only make jumps as illustrated in Figure 1 and similarly for the $B$ and $B^{\prime}$ cations. At the same time, for the oxygen vacancies, we have now the condition that the majority of them are involved in an independent vacancy diffusion mechanism. In our further discussion we will consider only oxygen vacancies involved in the cluster mechanism. For example, $R_{O}$ will be used for the oxygen (collective) displacement due to the cluster movement only, without contribution from the movements of the single oxygen vacancies and so on.

We introduce phenomenological coefficients $L_{A A}, L_{A A^{\prime}}, L_{A^{\prime} A^{\prime}}, L_{B B}, L_{B B^{\prime}}, L_{B^{\prime} B^{\prime}}$ and $L_{O O}$ and their correlated parts, the collective correlation factors $f_{i j}{ }^{(j)}$ for the diffusion of cations $A$ and $B$ and oxygen by way of the postulated cluster diffusion mechanism:

$$
L_{i j}=f_{i j}^{(j)} L_{j j}^{(0)},
$$

where the superscript (0) always indicates an uncorrelated part.
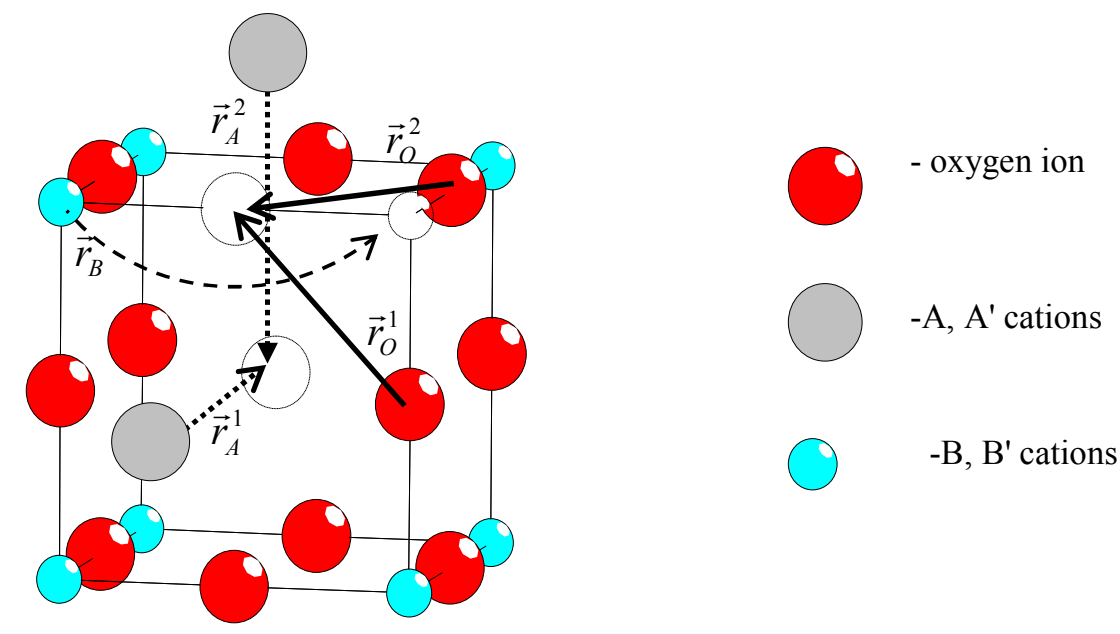

Figure 1. Schematic representation of the possible jumps of the vacancies in the cluster in the cubic perovskite structure. Unfilled circles represent vacancies.

The Einsteinian expression for the phenomenological coefficients is [5]:

$$
L_{i j}=\frac{<R_{i} \cdot R_{j}>}{6 V k T}
$$

where $R_{i}$ is the collective displacement of species $i$ in the volume $V$ in time $t$ and $k$ and $T$ are Boltzmann constant and temperature respectively. It is a natural requirement of the vacancycluster mechanism that the cation and anion vacancies have the same displacement after some long time $t$. Accordingly, the collective displacements of the cations and anions must therefore be equal, i.e. $R_{A}+R_{A^{\prime}}=R_{B}+R_{B^{\prime}}=R_{O}$. We then have a number of consequential relations between the phenomenological coefficients such as:

$$
L_{i A}+L_{i A^{\prime}}=L_{i B}+L_{i B^{\prime}},
$$


where $i=\mathrm{A}, \mathrm{A}^{\prime}, \mathrm{B}, \mathrm{B}^{\prime}, \mathrm{O}$. Assuming random mixing of the cations $\mathrm{A}$ and $\mathrm{A}^{\prime}$ and also of $\mathrm{B}$ and $\mathrm{B}^{\prime}$ and using exactly the same approach as given by Moleko and Allnatt [6] see also Allnatt and Lidiard [7] and Eq. 1, we soon have the following Sum-rule relations amongst the phenomenological coefficients:

$$
\begin{gathered}
2 L_{O O}^{\prime}+\frac{w_{O}}{w_{A}} L_{A O}+\frac{w_{O}}{w_{A^{\prime}}} L_{A^{\prime} O}+2 \frac{w_{O}}{w_{B}} L_{B O}+2 \frac{w_{O}}{w_{B^{\prime}}} L_{B^{\prime} O}=2 L^{\prime}(0) \\
L_{i i}+\sum_{j \neq i} \frac{w_{i}^{\prime}}{w_{j}^{\prime}} L_{i j}+\frac{w_{i}^{\prime}}{w_{O}} L_{i O}=L_{i i}^{(0)} \quad i, j=A, A^{\prime}, B, B^{\prime}
\end{gathered}
$$

Here $w_{A}^{\prime}=2 w_{A}, w_{A^{\prime}}^{\prime}=2 w_{A^{\prime}}$ and $w_{i}^{\prime}=w_{i}$ if $i \neq A, A^{\prime}, w_{\mathrm{O}}$ is the exchange frequency of a vacancy (of the vacancy-cluster) with an oxygen ion and $w_{\mathrm{A}}, w_{\mathrm{A}^{\prime}}$ and $w_{\mathrm{B}}, w_{\mathrm{B}^{\prime}}$ are the exchange frequencies of the cations with vacancies in the vacancy-cluster. $L_{\mathrm{OO}}^{\prime}$ denotes only that part of the $L_{\mathrm{OO}}$ which consists of the oxygen ions jumps in the cluster mechanism. If we make the sound assumption that $w_{\mathrm{O}}$ is much higher than the other atomic - cluster exchange frequencies we will have seven equations (any three from Eqs. 3 and four from Eqs. 4) to determine ten collective correlation factors (therefore an additional three will be needed for the complete analysis). The expressions for the uncorrelated parts $L_{\mathrm{ii}}{ }^{(0)}$ of the phenomenological coefficients can be found by inspection as:

$$
L_{i i}^{(0)}=\frac{z_{i} r_{i}^{2} c_{i} w_{i} c_{V i} N_{i}}{6 V k T}
$$

where the 'jump' coordination numbers are $z_{O}=2, z_{A}=2, z_{B}=1$.

In [4] using standard procedures [8,9] we performed Monte Carlo calculations of the collective correlation factors for the undoped oxide $\left(c_{A^{\prime}}=c_{B^{\prime}}=0\right)$ as functions of the ratio of exchange frequencies $w_{A} / w_{O}$ and $w_{B} / w_{O}$. It was found that there is perfect agreement between the Monte Carlo computer simulation results and the theoretical sum-rule expressions (see Figure 2). We assume that the Sum-rule expression is exact for the case of mixed/doped oxide (LSGM) as well.

\section{Tracer Diffusion}

In this section, we analyze limits on the ratios of the tracer diffusivities of $\mathrm{La}, \mathrm{Sr}$ and $\mathrm{Mg}$ in LSGM. In [3] it was found that all these tracer coefficients are almost identical (within about a factor of 3-4 if experimental error is taken into account).

In [4] it was shown that it is possible to derive an approximate theory for calculating tracer correlation factors. Unfortunately, the accuracy of this approximation is not sufficient to analyze the limits for the ratios of the tracer diffusion coefficients. Accordingly, we will test these limits by making use of Monte Carlo computer simulations. 


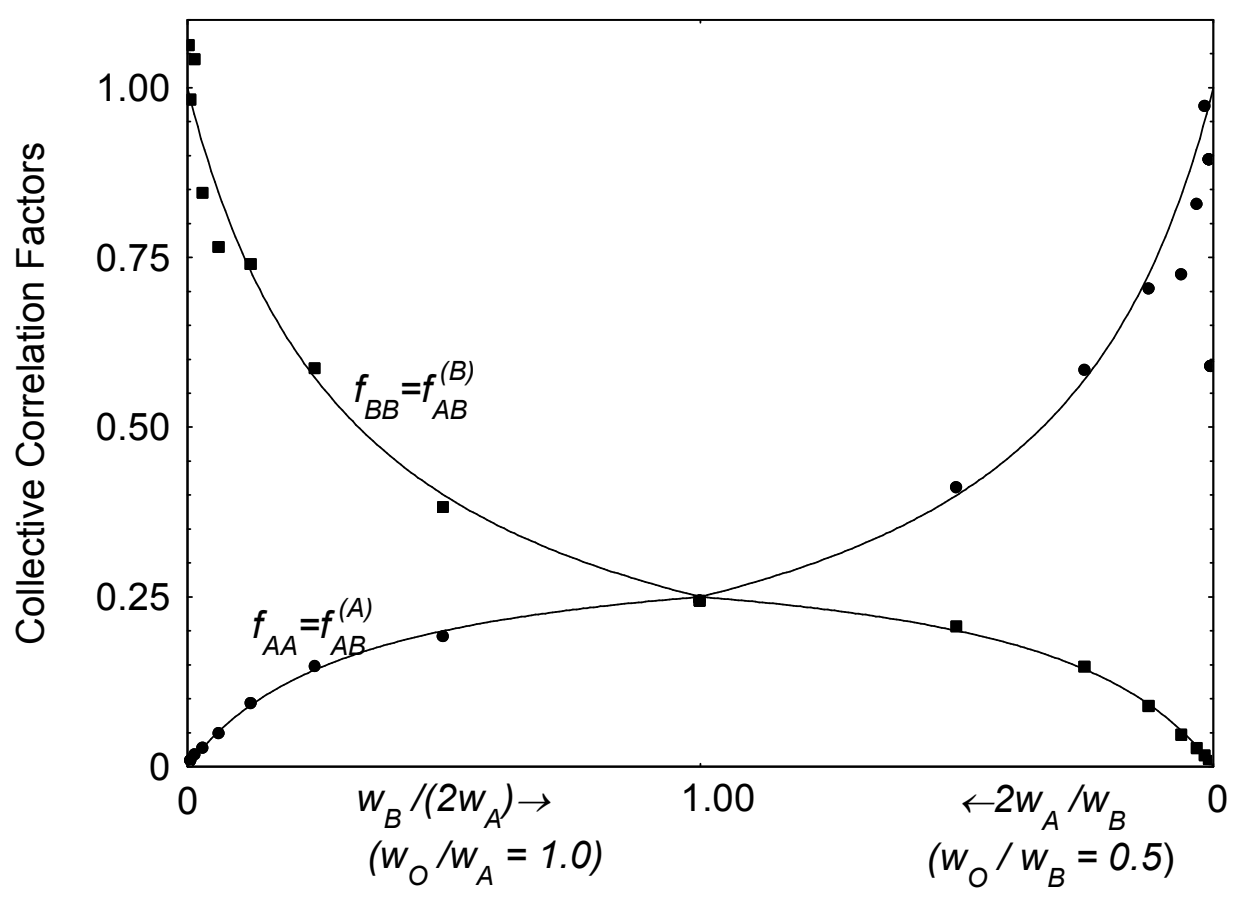

Figure 2. Collective correlation factors as functions of the ratios of the exchange frequencies. Symbols - Monte Carlo simulation results, lines - analytical relations derived on the basis of the Sum-rule.

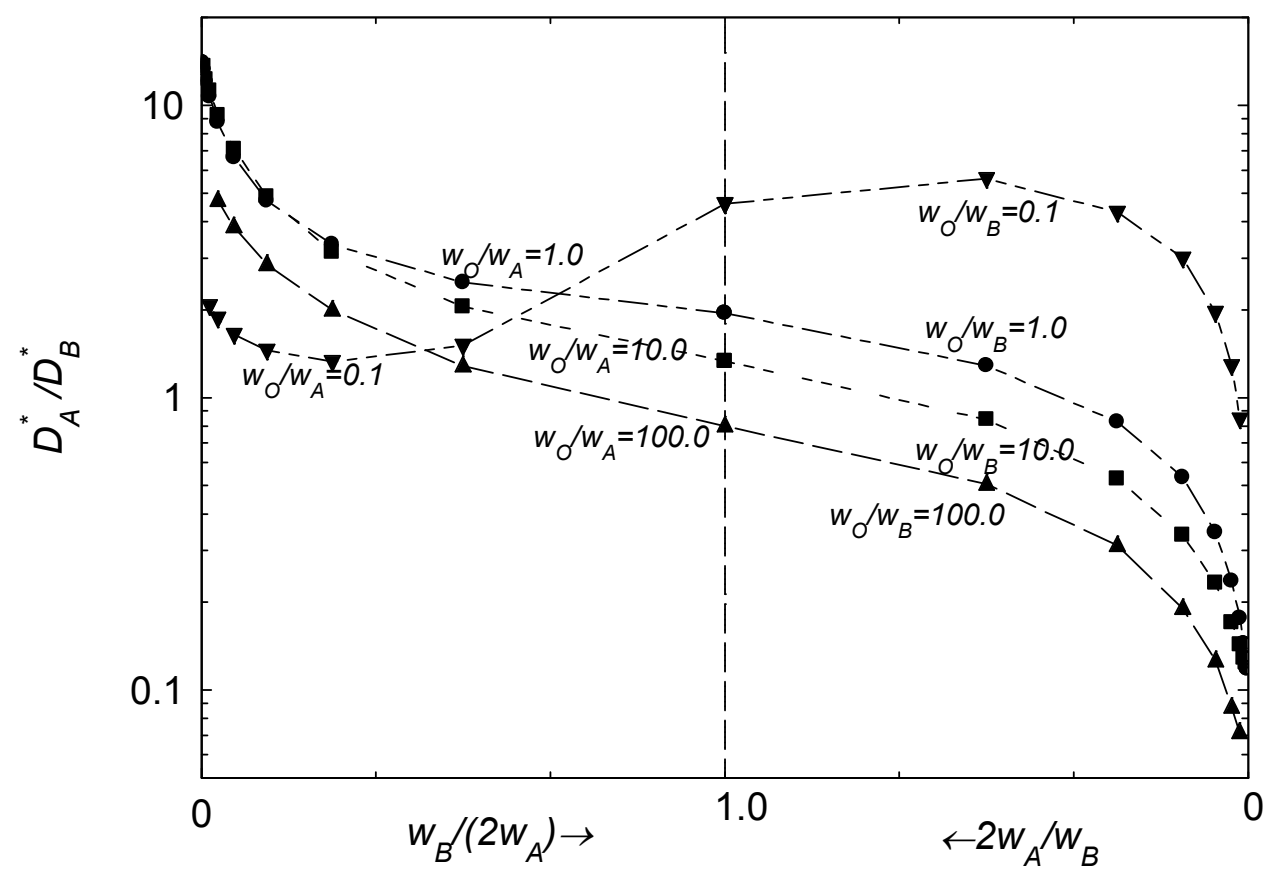

Figure 3. Ratio of the cation tracer diffusivities as a function of the ratios of the exchange frequencies as calculated by Monte Carlo simulations.

In order to calculate the tracer (or self) correlation factors we use similar Einsteinian expression as Eq. 2 but now in terms of the individual displacement $r_{j}$ of a tracer atom of type $j$ and the corresponding average number of jumps of the atom $m_{j}$ in time $t[8,10]$ :

$$
f_{j}=\frac{<r_{j}^{2}>}{m_{j} r_{j}^{2}}
$$


It was shown in [4] that the geometric tracer correlation factor $f_{0}$ for the cation sublattices when $w_{\mathrm{O}}$ goes to infinity, extrapolates to 0.654 , the value for diffusion by isolated vacancies in the s.c. lattice. In Fig. 3 we present results of calculations of the ratio of the tracer diffusion coefficients $D_{A}{ }^{*} / D_{B}{ }^{*}$ as functions of the ratios of the exchange frequencies (undoped oxide [4]). It is seen that the maximum of this ratio is slightly under fourteen (this is analogous to the behaviour of the vacancy-pair mechanism in the $B 2$ structure), whereas the maximum of $D_{B}{ }^{*} / D_{A}{ }^{*}$ is likely to be well under ten.

(a)

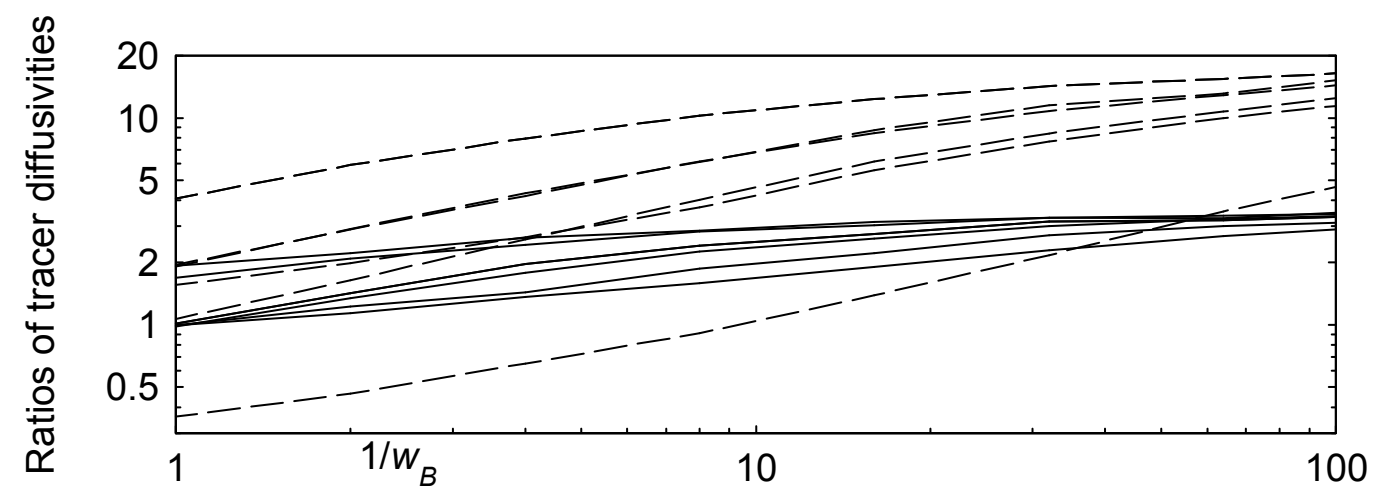

(b)

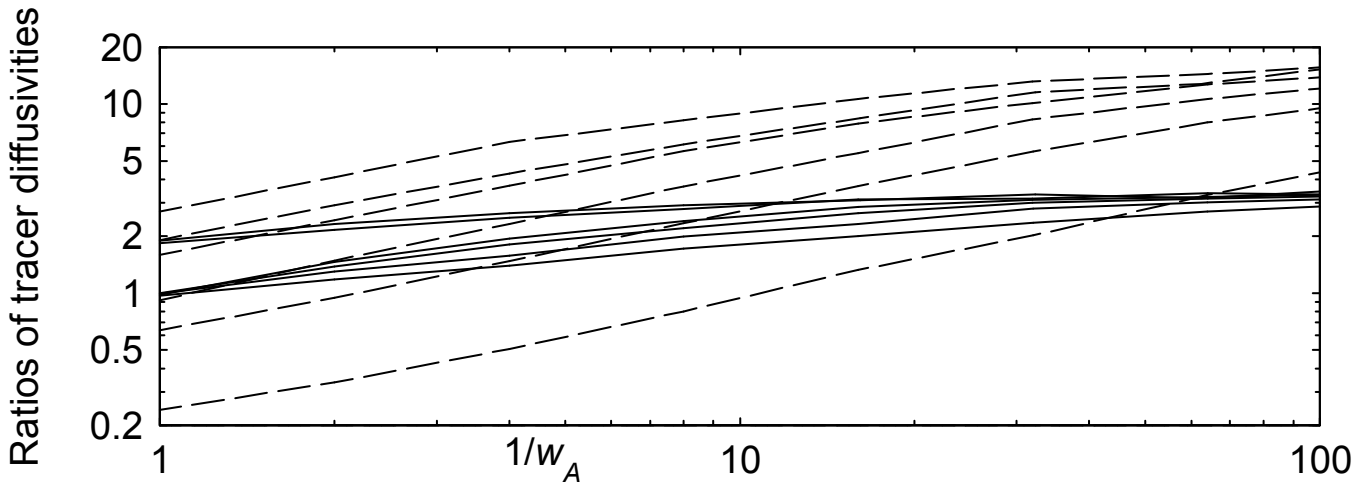

Figure 4. Ratios of tracer diffusivities: a) $D_{A}{ }^{*} / D_{B}{ }^{*}$ (dashed lines) and $D_{B^{\prime}}{ }^{*} / D_{B}{ }^{*}$ (solid lines); b) $D_{B}{ }^{*} / D_{A}{ }^{*}$ (dashed lines) and $D_{A^{\prime}}{ }^{*} / D_{A}{ }^{*}$ (solid lines). Set of frequencies used in a): for $w_{A}=w_{A^{\prime}}=w_{B^{\prime}}$ $=1.0$ there are two cases of $w_{\mathrm{O}}=1.0$ and 10.0; for $w_{A}=1.0, w_{A^{\prime}}=w_{B^{\prime}}=10.0$ there are two cases of $w_{\mathrm{O}}=10.0$ and 100.0; for $w_{B^{\prime}}=1.0$ there are two cases $\left\{w_{A}=w_{A^{\prime}}=w_{\mathrm{O}}=10.0\right\}$ and $\left\{w_{A}=w_{A^{\prime}}=\right.$ $\left.0.1, w_{\mathrm{O}}=1.0\right\} ; \mathrm{b}$ ): for $w_{B}=w_{A^{\prime}}=w_{B^{\prime}}=1.0$ there are two cases of $w_{\mathrm{O}}=1.0$ and 10.0 ; for $w_{B}=1.0$, $w_{A^{\prime}}=w_{B^{\prime}}=10.0$ there are two cases of $w_{\mathrm{O}}=10.0$ and 100.0; for $w_{A^{\prime}}=1.0$ there are two cases $\left\{w_{B}\right.$ $\left.=w_{B^{\prime}}=w_{\mathrm{O}}=10.0\right\}$ and $\left\{w_{B}=w_{B^{\prime}}=0.1, w_{\mathrm{O}}=1.0\right\}$.

When the doped oxide is considered (Figure 4) we need to take into account that for the composition of $c_{A^{\prime}}=c_{B^{\prime}}=0.1$ of the additional dopants on each cation sublattice the resulting (or average) cation vacancy exchange frequency will be almost unaffected by the (possibly) different mobility of the added element. This will mean that the general limits of the ratios of the tracer diffusivities even in the doped oxide can be expected to follow the behaviour in the un-doped oxide considered here.

In Figure 4 we present results of Monte Carlo simulations for the ratios of the tracer diffusivities for a number of combinations of exchange frequencies. These were chosen to cover the most likely cases. In the choices we assume primarily that the minority atomic components ( $\mathrm{Sr}\left(\mathrm{A}^{\prime}\right)$ and $\mathrm{Mg}\left(\mathrm{B}^{\prime}\right)$ ) are more mobile than the majority atomic components ( $\mathrm{La}(\mathrm{A})$ and $\mathrm{Ga}(\mathrm{B})$ ). This assumption can be justified by the tendency that has been observed in YSZ: the bigger the ion the more mobile it is on its sublattice [16]. In Fig. 4a we present cases where $w_{B}$ is varied 
from 1.0 to 0.01 and we plot ratios $D_{A}{ }^{*} / D_{B}{ }^{*}$ and $D_{A^{\prime}}{ }^{*} / D_{B}{ }^{*}$ as dashed lines and ratio $D_{B^{\prime}}{ }^{*} / D_{B}{ }^{*}$ as solid lines. In Figure $4 \mathrm{~b}$ we present the case where $w_{A}$ is varied from 1.0 to 0.01 and we plot ratios $D_{B}{ }^{*} / D_{A}{ }^{*}$ and $D_{B^{\prime}}{ }^{*} / D_{A}{ }^{*}$ as dashed lines and ratio $D_{A^{\prime}}{ }^{*} / D_{A}{ }^{*}$ as solid lines. We can see that the ratios of diffusivities of the atomic components occupying the same sublattice is always well under factor of 3.0 (this is only for the case when the minority component is similar or more mobile than the majority component: $\left.w_{B^{\prime}\left(A^{\prime}\right)} \geqslant w_{B(A)}\right)$. This is consistent with the experimental finding that $D_{L a}{ }^{*}$ and $D_{S r}{ }^{*}$ are almost identical. If we now check what would be the limits for this ratio of tracer diffusivities for the single vacancy mechanism on the cation sublattices we find using the Manning/MAA formalism that this is exactly the same result for the simple cubic lattice where the composition $c_{B^{\prime}\left(A^{\prime}\right)}=0.1$ and $w_{B^{\prime}\left(A^{\prime}\right)} \geqslant w_{B(A)}$ : the limit of the ratio of tracer diffusivities is about 3.0. Therefore the main evidence for the existence of the cluster mechanism in LSGM is the closeness of the diffusivities $D_{M g}{ }^{*}$ and $D_{L a}{ }^{*}$. These atoms are very different chemically and in size and therefore to show almost equal diffusivities over wide temperature range (about $500^{\circ}$ ) the diffusivities must be closely coupled, i.e. a cluster mechanism. In the following sections, we will assume that only the cluster mechanism or variations of the cluster mechanism is possible in LSGM.

\section{Critical Experiments for LSGM and YSZ}

To obtain more information about the diffusion mechanism in YSZ and LSGM one can conceive of two types of collective diffusion experiments: a kinetic demixing experiment in an electric field (and an oxygen pressure gradient for reference) and an interdiffusion experiment. Both experiments are, of course, very difficult because of the low diffusivities of the cation components. It will be important in the demixing experiment to detect any tendency for the samples to move as well as any tendency of the cations to produce demixed atomic concentration profiles.

The demixing type of experiment has been analyzed theoretically in detail for the case of YSZ.

Single vacancy mechanism in YSZ. We consider open system conditions where the system consists of fine grains with enough short circuits for the vacancies everywhere to very quickly establish equilibrium according to the external oxygen partial pressure [11]. For the case when only an electric field driving force is applied we have for the $\mathrm{Y}$ composition profile at steady state:

$$
\frac{d c_{Y}}{d \xi}=\frac{c_{Z r} c_{Y}\left(3 w_{Y 1}-4 w_{Z r 1}\right)}{c_{Y} w_{Z r 1}+c_{Z r} w_{Y 1}} \frac{e E d}{k T}
$$

In [1] it was shown that the demixing effect for the open system is much smaller than for the corresponding closed system.

Vacancy-pair mechanism in YSZ. When only an electric field is applied and for open system conditions, then for the $\mathrm{Y}$ atoms composition we have the following equation at steady state:

$$
\frac{d c_{Y}}{d \xi}=\frac{c_{Z r} c_{Y}\left(1+c_{Z r}\right)\left(w_{Y 1}-2 w_{Z r 1}\right)}{\left(1+2 c_{Z r}\right) c_{Y} w_{Z r 1}+\left(1+c_{Z r}-c_{Y}\right) c_{Z r} w_{Y 1}} \frac{e E d}{k T}
$$

For electric fields of the same strength we find that the demixing effect for the vacancy-pair mechanism (estimated with the parameters for the YSZ at 1573K) is about a factor of 3.5 smaller than for the single vacancy mechanism. Therefore if only one oxygen vacancy is involved in the diffusion mechanism in YSZ the result on the demixing profile is to make it significantly flatter, meaning that the demixing effect would be less noticeable. 
Vacancy-triplet mechanism in YSZ. When only an electric field is applied and for open system conditions, for the $\mathrm{Y}$ composition we have the following equation at steady state:

$$
\frac{d c_{Y}}{d \xi}=-\frac{c_{Z r} c_{Y}\left(1+c_{Z r}\right) w_{Y 1}}{\left(1+3 c_{Z r}\right) c_{Y} w_{Z r 1}+\left(1+c_{Z r}-2 c_{Y}\right) c_{Z r} w_{Y 1}} \frac{e E d}{k T}
$$

For the vacancy-triplet diffusion mechanism (with two oxygen vacancies involved) the result of the demixing experiment will be as if the cations were negatively charged (abnormal demixing effect) - due to the dragging effect of the two oxygen vacancies. This will result in the whole sample moving in the direction opposite to its natural (expected) movement in the electric field. The demixing prediction with the parameters for the YSZ at $1573 \mathrm{~K}$ is that it will be 4 times smaller than for the case of the same electric field strength and the single vacancy mechanism operating.

Cation vacancy-pair cluster mechanism in LSGM. For the cluster mechanism with only a cation vacancy-pair (two cation vacancies) operating in this oxide we can do a preliminary analysis as follows. With the Sum-rule relations described above for the mixed oxide and using the steady-state relations for the demixing experiment (using a notation similar to that used in [1]):

$$
J_{\mathrm{i}}-v c_{\mathrm{i}} N=0, \quad i=\mathrm{La}, \mathrm{Sr}, \mathrm{Ga}, \mathrm{Mg}
$$

we will have that $\left(F_{i}=q_{i} e E, q_{\mathrm{La}}=q_{\mathrm{Ga}}=+3, q_{\mathrm{Ga}}=q_{\mathrm{Mg}}=+2 ; q_{\mathrm{O}}=-2\right)$

$$
\nabla \mu_{i}-F_{i}=-\frac{v}{c_{V} 2 w_{i} a^{2}}, \quad \nabla \mu_{j}-F_{j}=-\frac{v}{c_{V} w_{j} a^{2}} \quad i=\mathrm{La}, \mathrm{Sr} ; j=\mathrm{Ga}, \mathrm{Mg}
$$

With open system conditions we will soon have that at steady state:

$$
\begin{aligned}
& \frac{d c_{S r}}{d \xi}=\frac{c_{L a} c_{S r}\left(2 w_{S r}-3 w_{L a}\right)}{c_{L a} w_{S r}+c_{S r} w_{L a}} \frac{e E d}{k T} \\
& \frac{d c_{M g}}{d \xi}=\frac{c_{G a} c_{M g}\left(2 w_{M g}-3 w_{G a}\right)}{c_{G a} w_{M g}+c_{M g} w_{G a}} \frac{e E d}{k T}
\end{aligned}
$$

Assuming that in the demixing experiment a correct tendency towards the steady-state solution can be observed, we can determine (on the basis of the preferential demixing on the cation sublattice) if there are significant differences between the cation mobilities $\left(3 w_{\mathrm{La}}, 2 w_{\mathrm{Sr}}\right)$ and $\left(3 w_{\mathrm{Ga}}, 2 w_{\mathrm{Mg}}\right)$. Of great help could be results of the oxygen pressure gradient only applied to the same sample (if possible). This would give additional information on the differences between $w_{\mathrm{La}}, w_{\mathrm{Sr}}$ for one cation sublattice and $w_{\mathrm{Ga}}, w_{\mathrm{Mg}}$ for the other cation sublattice.

Vacancy-triplet cluster mechanism in LSGM. In this variation of the cluster mechanism we assume that two cation vacancies and one oxygen vacancy are involved into the cluster movement. This case is extremely complicated for a very general set of exchange frequencies. To simplify the resulting expressions we assume that $w_{\mathrm{La}} \approx w_{\mathrm{Ga}}$ and $w_{\mathrm{Sr}} \approx w_{\mathrm{Mg}}$. Then, following the same arguments as for the cation vacancy-pair mechanism analyzed above we will have that: 


$$
\begin{gathered}
\nabla \mu_{i}+0.5 \nabla \mu_{O}-F_{i}-0.5 F_{O}=-\frac{v}{c_{V} 2 w_{i} a^{2}}, \quad \nabla \mu_{j}+0.5 \nabla \mu_{O}-F_{j}-0.5 F_{O}=-\frac{v}{c_{V} w_{j} a^{2}} \\
i=\mathrm{La}, \mathrm{Sr} ; j=\mathrm{Ga}, \mathrm{Mg}
\end{gathered}
$$

With open system conditions we will soon have that at steady state:

$$
\begin{aligned}
\frac{d c_{S r}}{d \xi} & =\frac{c_{L a} c_{S r} c_{O}\left(w_{S r}-2 w_{L a}\right)}{c_{L a}\left(2 c_{O}-c_{S r}\right) w_{S r}+c_{S r}\left(2 c_{O}+c_{L a}\right) w_{L a}} \frac{e E d}{k T} \\
\frac{d c_{M g}}{d \xi} & =\frac{c_{G a} c_{M g} c_{O}\left(w_{M g}-2 w_{G a}\right)}{c_{G a}\left(2 c_{O}-c_{M g}\right) w_{M g}+c_{M g}\left(2 c_{O}+c_{G a}\right) w_{G a}} \frac{e E d}{k T}
\end{aligned}
$$

We note that it will be impossible, using only a demixing experiment as described above, to differentiate between the vacancy-pair mechanism and vacancy-triplet mechanisms as variations of the vacancy cluster mechanism. It is similar to the YSZ situation with the single vacancy and vacancy-pair mechanisms. In the case of LSGM it seems that no more than one oxygen vacancy can be involved in the cluster mechanism. Therefore an abnormal demixing effect is less expected.
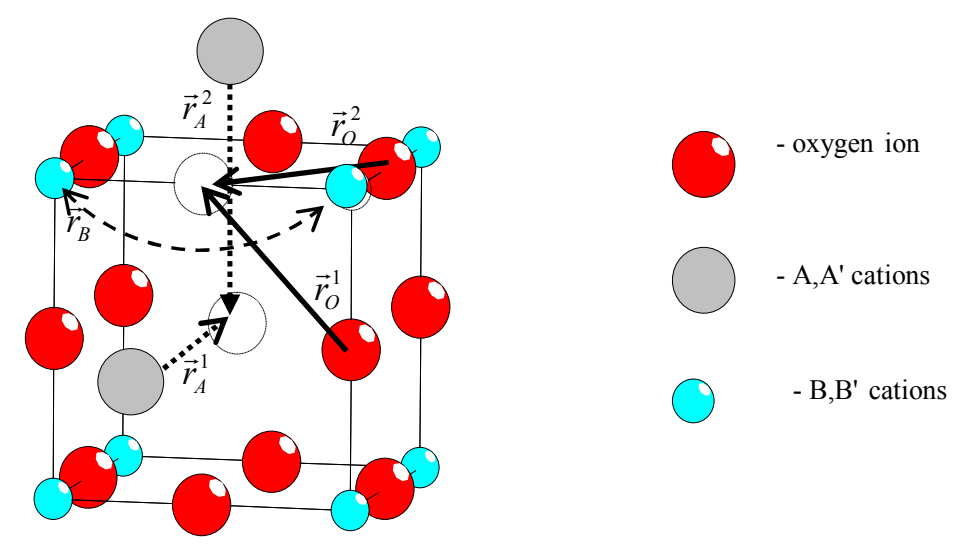

Figure 5. Schematic representation of a variation of the vacancy cluster mechanism jumps (Figure 1) where only one cation vacancy (on A sublattice) is involved, the diffusion mechanism on the $B$ sublattice is equivalent to a direct atomic exchange mechanism. The total result will also be equivalent to a six-jump-cycle mechanism with a vacancy starting on A sublattice. Unfilled circles represent vacancies.

Cluster mechanism with only one cation vacancy in LSGM. This is a variation of the cluster mechanism with only one cation vacancy that mainly resides on the La sublattice and possibly one or more oxygen vacancies. Some evidence for this mechanism can be drawn from [3] and from a later molecular dynamics simulation [16] that confirms the existence of B cations on A sublattice (anti-site defects). For this case, with one or more oxygen vacancies 'support' from the oxygen sublattice we can see that atoms on the Ga sublattice can only move around using the same vacancy. Then the resulting effect corresponds to the direct atomic exchange mechanism of the atoms on the Ga sublattice (see Figure 5).

If this mechanism is the main diffusion mechanism for bulk diffusion then obviously there will not be any demixing effect in the bulk: at least no detectable movements, but it is still possible to have some atomic segregation at opposite sides of grains.

The conclusion can be made that the demixing experiment (being very difficult because of the very low bulk atomic mobilities in LSGM) is not likely to differentiate between the vacancy-pair 
and vacancy-triplet (or two cluster variations) mechanisms. On the other hand, if only one cation vacancy is involved in the cluster mechanism then there will not be any tendency for demixing inside the grains.

Interdiffusion experiments. In an interdiffusion experiment there is a real possibility to make a definitive set of experiments. In [7, 12-15] it was shown that the interdiffusion process on cation sublattice(s) in ionic materials depends strongly on the mobility of the anions (and vice versa). A similar argument is appropriate for the case of LSGM for the two cation sublattices that must affect interdiffusion processes of each other. Of course, because of the very high oxygen mobility for LSGM, the interdiffusion processes on the cation sublattices are not affected by the movement of the anions. For the single vacancy mechanism there should not be any mutual dependence of the interdiffusion processes on the two cation sublattices. But for the cluster mechanism to produce the same effect it is important if there are 'similar' interdiffusion processes happening at the same time on both sublattices (details of the analysis will be presented elsewhere). Accordingly, we suggest consideration of the following interdiffusion couples: 1: $\left(\mathrm{La}_{0.9} \mathrm{Sr}_{0.1} \mathrm{Ga}_{0.9} \mathrm{Mg}_{0.1} \mathrm{O}_{2.9}\right)$ - $\left(\mathrm{La}_{0.8} \mathrm{Sr}_{0.2} \mathrm{Ga}_{0.8} \mathrm{Mg}_{0.2} \mathrm{O}_{2.8}\right)$ - this is a 'reference experiment'; 2: $\left(\begin{array}{llll}\mathrm{La} & \mathrm{Ga}_{0.9} & \mathrm{Mg}_{0.1} & \mathrm{O}_{2.95}\end{array}\right)$ - ( $\left.\mathrm{La} \mathrm{Ga}_{0.8} \mathrm{Mg}_{0.2} \mathrm{O}_{2.9}\right)$; 3: $\left(\mathrm{La}_{0.9} \mathrm{Sr}_{0.1} \mathrm{Ga} \mathrm{O}_{2.95}\right)$ - $\left(\mathrm{La}_{0.8} \mathrm{Sr}_{0.2} \mathrm{Ga} \mathrm{O}_{2.9}\right)$. Couple 1 represents the case for both sublattices to undergo the interdiffusion process and can be expected to show if there are any real differences between mobilities of the cations on the same sublattice: $\mathrm{La}$ and $\mathrm{Sr}$ and $\mathrm{Ga}$ and $\mathrm{Mg}$. If there are differences between mobilities of the cations on the same sublattice then couples 2 and 3 might show a difference in the interdiffusion concentration profiles (on the individual sublattice) compared to couple 1. If there are obvious differences (e.g. much less developed profiles for the same interdiffusion times) then this would be definitive proof of the cluster mechanism.

We can also suggest a further couple (couple 4): $\left(\mathrm{La}_{0.9} \mathrm{Sr}_{0.1} \mathrm{Ga}_{0.8} \mathrm{Mg}_{0.2} \mathrm{O}_{2.85}\right)-\left(\mathrm{La}_{0.8} \mathrm{Sr}_{0.2}\right.$ $\mathrm{Ga}_{0.9} \mathrm{Mg}_{0.1} \mathrm{O}_{2.85}$ ) - this couple has the same oxygen concentration on both sides. It could well be the most interesting couple to study (couple 1 should be analyzed as a reference point). Couple 4, in principle, might produce the enhanced combined results of couples 2 and 3.

\section{Summary}

In this paper, we have discussed different possible cation diffusion mechanisms in YSZ and LSGM. Monte Carlo simulations were reported of tracer diffusivities in LSGM for a postulated cluster mechanism. These simulations extended earlier simulations on un-doped material. The limits of the ratio of the diffusivities were consistent with experimental tracer diffusion findings over a wide range of cation-vacancy exchange frequencies. We also developed relationships between the phenomenological coefficients and used these relationships to predict possible experimental demixing and interdiffusion outcomes in YSZ and LSGM.

\section{Acknowledgements}

We wish to thank the Australian Research Council for its support of this work under the Discovery Project Grants and Linkage Grants Scheme.

\section{References}

[1] I V Belova, D Samuelis, M Martin and G E Murch: Phil. Mag. Vol. 87 (2007), p. 1447.

[2] O. Schulz and M. Martin: Solid State Ionics Vol. 135 (2000), p. 549.

[3] O. Schulz, M. Martin, Ch. Argirusis and G. Borchardt: Phys. Chem. Chem. Phys. Vol. 5 (2003), p. 2308.

[4] I.V. Belova, D. Samuelis, M. Martin and G.E. Murch: Defect and Diffusion Forum Vol. 263 (2007), p. 81.

[5] A.R. Allnatt: J. Phys. C: Solid St. Phys. Vol. 15 (1982), p. 5605.

[6] L.K. Moleko and A.R. Allnatt: Phil. Mag. A 58 (1988), p. 677. 
[7] A.R. Allnatt and A.B. Lidiard: Atomic Transport in Solids (Cambridge University Press, Cambridge 1993).

[8] A.R. Allnatt and E.L. Allnatt: Phil. Mag. A Vol. 49 (1984), p. 625.

[9] G.E. Murch: Diffusion in Crystalline Solids, edited by G.E. Murch and A.S. Nowick, Academic Press, Orlando Fl (1984), p.379.

[10] H.J. DeBruin and G.E. Murch: Phil. Mag. Vol. 27 (1973), p. 1475.

[11] I.V. Belova, M.J. Brown and G.E. Murch: Solid State Ionics Vol. 167 (2004), p. 175.

[12] R. Lindström: J. Phys. Chem. Solids Vol. 30 (1969), p. 401.

[13] R. Lindström: J. Phys. C Vol. 7 (1974), p. 3909.

[14] I.V. Belova and G.E. Murch: Phil. Mag. Vol. 84 (2004), p. 2139.

[15] I.V. Belova and G.E. Murch: Phil. Mag. Vol. 84 (2004), p. 3637.

[16] M. Kilo, M.A Taylor, C. Argirusis, G. Borchardt, R.A. Jackson, O. Schulz, M. Martin, M. Weller: Solid State Ionics Vol. 175 (2004), p. 823 